\title{
Disentangling Vector-Borne Transmission Networks: A Universal DNA Barcoding Method to Identify Vertebrate Hosts from Arthropod Bloodmeals
}

\author{
Miguel Alcaide ${ }^{1 *}$, Ciro Rico ${ }^{1}$, Santiago Ruiz ${ }^{2}$, Ramón Soriguer ${ }^{1}$, Joaquín Muñoz ${ }^{1}$, Jordi Figuerola ${ }^{1}$ \\ 1 Estación Biológica de Doñana (CSIC), Isla de la Cartuja, Sevilla, Spain, 2 Diputación de Huelva, Área de Medio Ambiente, Huelva, Spain
}

\begin{abstract}
Emerging infectious diseases represent a challenge for global economies and public health. About one fourth of the last pandemics have been originated by the spread of vector-borne pathogens. In this sense, the advent of modern molecular techniques has enhanced our capabilities to understand vector-host interactions and disease ecology. However, host identification protocols have poorly profited of international DNA barcoding initiatives and/or have focused exclusively on a limited array of vector species. Therefore, ascertaining the potential afforded by DNA barcoding tools in other vector-host systems of human and veterinary importance would represent a major advance in tracking pathogen life cycles and hosts. Here, we show the applicability of a novel and efficient molecular method for the identification of the vertebrate host's DNA contained in the midgut of blood-feeding arthropods. To this end, we designed a eukaryote-universal forward primer and a vertebrate-specific reverse primer to selectively amplify 758 base pairs $(\mathrm{bp})$ of the vertebrate mitochondrial Cytochrome $c$ Oxidase Subunit I (COI) gene. Our method was validated using both extensive sequence surveys from the public domain and Polymerase Chain Reaction (PCR) experiments carried out over specimens from different Classes of vertebrates (Mammalia, Aves, Reptilia and Amphibia) and invertebrate ectoparasites (Arachnida and Insecta). The analysis of mosquito, culicoid, phlebotomie, sucking bugs, and tick bloodmeals revealed up to 40 vertebrate hosts, including 23 avian, 16 mammalian and one reptilian species. Importantly, the inspection and analysis of direct sequencing electropherograms also assisted the resolving of mixed bloodmeals. We therefore provide a universal and high-throughput diagnostic tool for the study of the ecology of haematophagous invertebrates in relation to their vertebrate hosts. Such information is crucial to support the efficient management of initiatives aimed at reducing epidemiologic risks of arthropod vector-borne pathogens, a priority for public health.
\end{abstract}

Citation: Alcaide M, Rico C, Ruiz S, Soriguer R, Muñoz J, et al. (2009) Disentangling Vector-Borne Transmission Networks: A Universal DNA Barcoding Method to Identify Vertebrate Hosts from Arthropod Bloodmeals. PLoS ONE 4(9): e7092. doi:10.1371/journal.pone.0007092

Editor: Laurent Rénia, BMSI-A*STAR, Singapore

Received July 3, 2009; Accepted August 26, 2009; Published September 21, 2009

Copyright: (c) 2009 Alcaide et al. This is an open-access article distributed under the terms of the Creative Commons Attribution License, which permits unrestricted use, distribution, and reproduction in any medium, provided the original author and source are credited.

Funding: This study was funded by the Consejería de Innovación, Ciencia y Empresa, Junta de Andalucía (Proyecto de Investigación de Excelencia P07-RNM02511) and partially funded by the EU grant GOCE-2003-010284 EDEN (http://www.eden-fp6project.net/); it is catalogued by the EDEN Steering Committee as EDEN0158. The contents of this publication are the sole responsibility of the authors and do not necessarily reflect the views of the European Commission.

Competing Interests: The authors have declared that no competing interests exist.

* E-mail: malcaide@ebd.csic.es

\section{Introduction}

The control of emerging infectious diseases constitutes one of the most important concerns of global economies and human health. Recent studies have emphasized that the majority of the last pandemics have been originated by zooneses in the wild. The participation of vectors in the spread of zoonotic diseases was estimated to occur in about one fourth of pathogen outbreaks during the last century [1]. Importantly, human related activities are contributing to increase the impact of vector-borne diseases by increasing vector density (e.g. irrigation, urbanization, dam construction) or by introducing pathogens into areas in which they had been hitherto absent [2]. As a result, there has been the need to control the population of blood-feeding arthropods to reduce epidemiologic risks because they may harbor pathogens responsible for serious infectious diseases such as malaria, viral encephalitis, West Nile virus, Chagas disease, Lyme disease or African sleeping sickness. Although pathogen prevalence in vectors is usually low, a correct understanding of vector-hosts interactions is crucial to predict transmission patterns and for the development of efficient control policies.
The enhancement of species identification since the advent of molecular methods has revolutionized our view of such complex ecological networks at the same time that it has contributed to gain insights on the co-evolutionary relationships between vectors, pathogens and their hosts [reviewed in 3]. The emerging of international initiatives such as the Consortium for the Barcode of Life (www.barcoding.si.edu) is expected to greatly expand the benefits of molecular methods in this field. The DNA barcoding project aims to establish a 648-bp fragment of the Cytochrome $c$ Oxidase Subunit I (COI) mitochondrial gene as universal method for the taxonomic classification of biodiversity $[4,5]$. The coverage provided by barcoding databases will soon facilitate an accurate assignment of host-vector associations, and their use by ecologists and epidemiologists is believed to increase considerably during the next few years [6]. One of the most important advantages of the COI locus to identify vertebrate hosts is linked to its model of molecular evolution, which is believed to provide better resolution of deeper taxonomic affinities than other molecular markers $[7,8]$. In this sense, precise host identifications might be crucial given that pathogen outcomes, exposure to vectors, demographic 
parameters and dispersal patterns may vary considerably even between closely related species [9-11]. Nevertheless, identification methods based on the partial sequencing of the vertebrate COI gene from arthropod bloodmeals have been underutilized in relation to other molecular approaches [3]. In fact, COI identification protocols conducted to date have focused exclusively on mosquito bloodmeals $[3,12]$. There is therefore the need to ascertain and take advantage of the potential afforded by DNA barcoding tools in other vector-host systems of public health and veterinary importance.

In this study, we have designed a single pair of primers for the selective analysis of host COI sequences from arthropod bloodmeals. Our method was also aimed at accelerating host identification and reducing both laboratory efforts and costs.

\section{Results}

\section{Validation of vertebrate-specific PCR amplification}

Primers M13BC-FW and BCV-RV1 were validated using high quality DNA extracts from avian (lesser kestrel Falco naumanni, Spanish Imperial eagle Aquila adalberti), mammalian (water vole Arvicola sapinus), amphibian (natterjack toad Bufo calamita) and reptilian (Iberian wall lizard Podarcis hispanica) species. PCR experiments always yielded high concentration of amplicons of the expected size. The same PCR protocol failed to amplify the target fragment when applied to DNA extracts obtained from the abdomen of non-engorged arthropods (mosquitoes, ticks, culicoids and sandflies). Nonetheless, we successfully amplified and sequenced a fragment of the arthropod COI locus using the eukaryote-universal primers proposed for the 'DNA mini-barcoding' approach [13]. It is important to emphasize that eukaryoteuniversal primers preferentially amplified vector COI sequences when applied to mosquito bloodmeals. On the contrary, our vertebrate-specific primer set only replicated host COI genes.

\section{Assignment of unknown $\mathrm{COI}$ sequences replicated from bloodmeals to specific vertebrate hosts \\ The first PCR reaction with primers M13BC-FW and BCV-} RV1 yielded suitable concentrations of PCR products for sequencing in 43 out of 100 mosquito bloodmeals. Non-suitable samples for sequencing displayed either low concentration of PCR products or a lack of bands of the expected size. Nonetheless, a nested PCR using M13 and BCV-RV2 primers generated suitable positives for sequencing in 97 out of 100 mosquito bloodmeals. The nested-PGR also proved satisfactory when applied to the bloodmeals of additional vector species (ticks, sandflies, culicoids and blood-sucking bugs).

The bioinformatics platform supported by the Barcode of Life Data Systems (BOLD) database permitted us to identify the origin of the bloodmeals contained in the midgut of several species of mosquitoes and other blood-fed ectoparasites (see Table 1). This is an online workbench that aids collection, management, analysis, and use of DNA barcodes by researches in different fields (www. barcodinglife.org). The inspection of sequencing electropherograms supported the amplification of the mitochondrial barcode locus from one single vertebrate host in the vast majority of cases. The similarity of our unknown vertebrate COI sequences with respect to those from museum voucher specimens was always $>99 \%$ except for five cases. Various unknown vertebrate COI sequences could not be assigned at the species level. These COI sequences showed the highest sequence similarity $(>90 \%)$ with species of the genus Lepus, Lynx, Mus, Alectoris and the Family Herpestidae. Given that the unique members of the genus Lepus, Alectoris and the Family Herpestidae inhabiting the south of Spain are

Table 1. Vertebrate hosts for different species of blood-feeding ectoparasites collected in South-western Spain.

\begin{tabular}{|c|c|c|}
\hline Species & Mammalian host & Avian host \\
\hline Anopheles algeriensis (Insecta: Culicidae) & Dama dama (1); Bos taurus (1) & \\
\hline Anopheles atroparvus (Insecta: Culicidae) & Bos taurus (3); Oryctolagus cuniculus (1) & \\
\hline Culex modestus (Insecta: Culicidae) & & $\begin{array}{l}\text { Anas platyrhynchos (2); Anser anser (1); Chen } \\
\text { caerulescens (1); Branta canadensis (1); Egretta garzetta } \\
\text { (1); Tadorna ferruginea (1); Anas strepera (1); Gallus } \\
\text { gallus (1); Ardea cinerea (1); Anas acuta (1); Tadorna } \\
\text { tadorna }\end{array}$ \\
\hline Culex perexiguus (Insecta: Culicidae) & Rattus norvergicus (1); Canis familiaris (1) & Alectoris rufa (1); Streptopelia decaocto (1) \\
\hline Culex pipiens (Insecta: Culicidae) & $\begin{array}{l}\text { Homo sapiens (1); Herpestes ichneumon (1); } \\
\text { Felis catus (1); Canis familiaris (2) }\end{array}$ & $\begin{array}{l}\text { Passer domesticus (7); Turdus merula (3); Streptopelia } \\
\text { decaocto (3); Galerida cristata (1); Sturnus vulgaris (2); } \\
\text { Cairina moschata (1); Grus grus (1); Sylvia melanocephala } \\
\text { (1); Alectoris rufa (1) }\end{array}$ \\
\hline Culex theileri (Insecta: Culicidae) & $\begin{array}{l}\text { Bos taurus (8); Cervus elaphus (4); Dama dama (2); Equus } \\
\text { caballus (3); Homo sapiens (1); Lepus granatensis (2); } \\
\text { Oryctola gus cuniculus (1); Sus scrofa (2) }\end{array}$ & Bubulcus ibis (1); Meleagris gallopavo (1) \\
\hline Ochlerotatus caspius (Insecta: Culicidae) & $\begin{array}{l}\text { Bos taurus (3); Canis familiaris (3); Capra hircus (1); Felis } \\
\text { catus (1); Cervus elaphus (1); Equus caballus ( } 2 \text {; } \\
\text { Oryctolagus cuniculus (2); Ovis aries (2); Sus scrofa (1) }\end{array}$ & $\begin{array}{l}\text { Gallus gallus (4); Podiceps nigricollis (1); Passer } \\
\text { domesticus (2); Turdus merula (1); Sturnus vulgaris (1) }\end{array}$ \\
\hline Culiseta longiareolata (Insecta: Culicidae) & & Passer domesticus (2) \\
\hline Phlebotomus sp. (Insecta: Phlebotominae) & Oryctolagus cuniculus (2) & \\
\hline Culicoides sp. (Insecta: Ceratopogonidae) & & Passer domesticus (2) \\
\hline Dipetalogaster maximus (Insecta: Reduviidae) & Lynx pardinus (2) & \\
\hline Rhipicephalus spp. (Arachnida: Ixodidae) & Mus spretus (1) Canis familiaris (2) & \\
\hline
\end{tabular}


not included within the BOLD System database yet, we deduced that these species could be the Iberian hare Lepus granatensis, the red-legged partridge Alectoris rufa and the Egyptian mongoose Herpestes ichneumon. Furthermore, we know that the two remaining COI sequences belonged to the Iberian Lynx Lynx pardinus and the Algerian mouse Mus spretus because ectoparasites were directly sampled on hosts. Overall, our molecular method allowed identifying 40 different vertebrate hosts, including 16 mammalian, 23 avian and one reptilian species (see Table 1). DNA extraction and PCR negative controls did not yield PCR bands and the analysis of our positive control always matched COI sequences of the mallard duck Anas platyrhynchos. Repeatability experiments performed over those bloodmeals reporting rare hosts (i.e. those found only in one bloodmeal) were also successful.

\section{Identification of mixed bloodmeals}

The alignment of ambiguous COI sequences (i.e. those displaying double peaks at different positions of sequencing electropherograms) with respect to our validated set of vertebrate COI sequences indicated four mixed bloodmeals. The inspection of sequencing electropherograms suggested the co-amplification of the COI locus from two different vertebrate hosts. A specimen of Ochlerotatus caspius and a specimen of Culex pipiens could have fed on a house sparrow Passer domesticus and a common blackbird Turdus merula (see Fig. 1). A specimen of Culex modestus could have fed on a common goose Anser anser and a domestic snow goose Chen caerulescens, and a specimen of Culex theileri could have fed on a red deer Cerous elaphus and a cow Bos taurus. In some cases, particularly for the wild boar Sus scrofa, we suspected that double peaks could be related to the co-amplification of nuclear insertions of the mitochondrial genome or heteroplasmy. We submitted unambiguous stretches of COI sequences to the public databases to ensure that these DNA sequences could only be originated from the same species. Then, sequence identities larger than 99\% were only reported with respect to the same, single vertebrate host.

\section{Discussion}

In this study, we describe a straightforward and universal method for the PCR specific amplification and analysis of the vertebrate barcode COI locus from the midgut of blood-feeding arthropods. The suitability of a single pair of primers capable to cope with all candidate hosts across a broad spectrum of vectors constitutes the major improvement put forward by our method. Although single pairs of primers were formerly available for the amplification of the vertebrate COI [12] and Cytochrome $b$ (Cyt $b$ ) genes [14], empirical support for their suitability in other vector
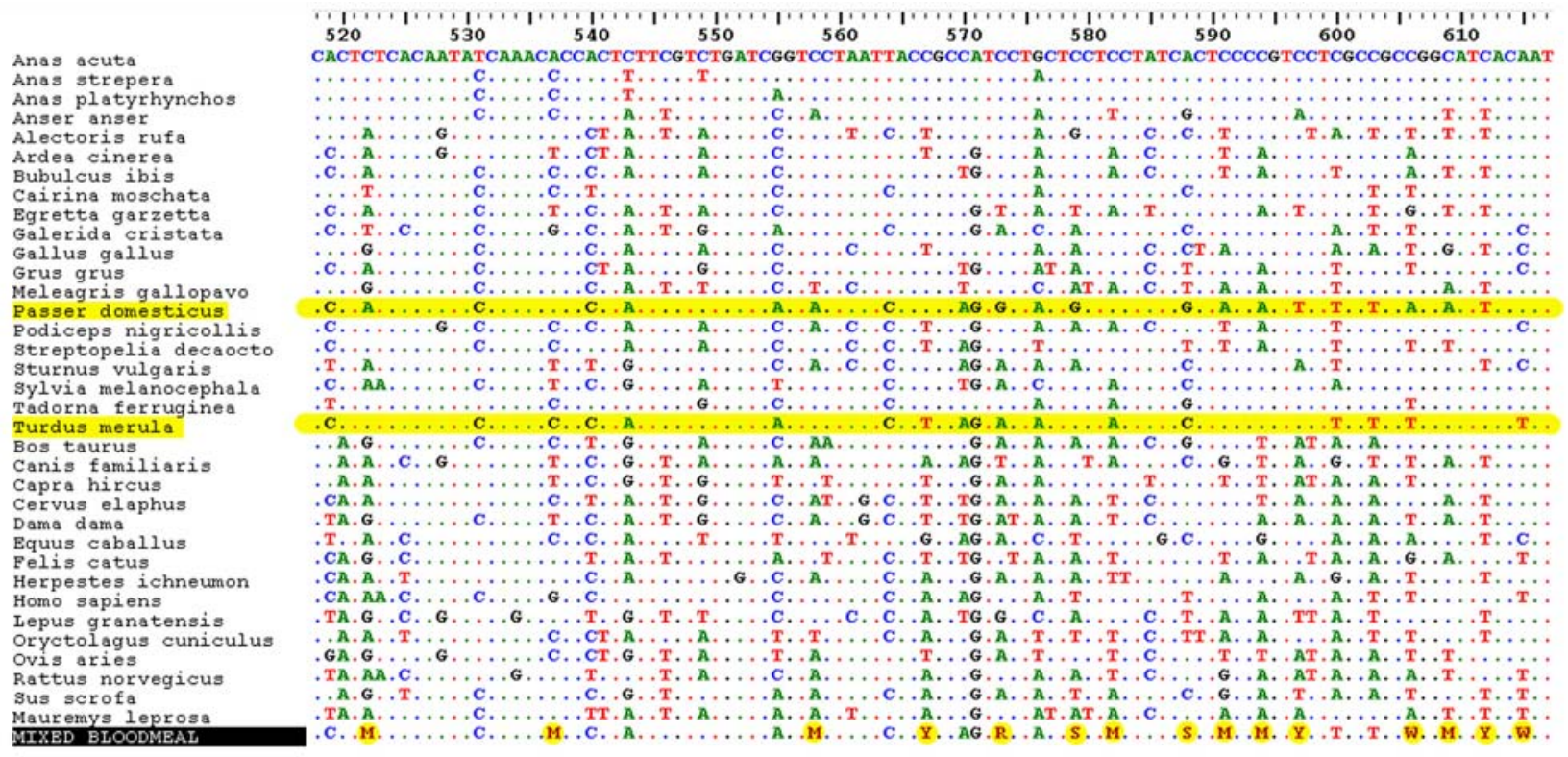

520

550

580

610

C C CTMTC ACAA TAC CAAACMCC C C TAT TC GT C TO ATC A GTMC TAAT CACYGCA GTRC TACT SCTMC TATC SCTMC CM GT YCT TGCTGCWG GMAT YACWA 1

Figure 1. Identification of a mixed bloodmeal. The co-amplification of $\mathrm{COI}$ fragments from different hosts may generate several ambiguous nucleotide positions (in yellow and indicated by arrows). The ambiguous sequence can be subsequently compared with respect to a validated set of $\mathrm{COI}$ sequences from the hosts typically found in the study area. In this example, the sequencing electropherogram obtained could be the result of the simultaneous amplification of DNA from house sparrow Passer domesticus and common blackbird Turdus merula.

doi:10.1371/journal.pone.0007092.g001 
species rather than mosquitoes and even the possible coamplification of invertebrate DNA, respectively, could be considered as major limitations. In fact, the vast majority of studies have employed cocktails of primers to deal with different vertebrate hosts [reviewed in 3]. The inconvenience of using avian or mammalian specific primers for those vectors traditionally considered ornithophilic or mammalphilic is also highlighted in the present study. As an example, we found that some mosquito species such as Culex pipiens, Cx. theileri and Ochlerotatus caspius can feed occasionally on other vertebrates groups (see Table 1). Shedding light on this kind of relationships would provide valuable information to understand pathogen transmission between different vertebrate groups.

One of the most interesting methodological advantages of our assay relies on the suitability of the inexpensive HotSHOT protocol for a rapid and feasible DNA extraction from bloodmeals. Success of our nested-PCR approach was $>90 \%$ for the bloodmeals analyzed, a similar value to other studies based on the amplification of a shorter fragment $(<400 \mathrm{bp})$ of the Cyt $b$ gene [e.g. 15]. Assuming a Taq DNA Polymerase error rate about $1 \times 10^{-4}$ base substitutions/bp/cycle, the total number of mutations introduced by our nested PCR protocol is around 8.24 base substitution per sequence (i.e. $1 \times 10^{-4} \times 758 \mathrm{bp} \times 110$ cycles $(75$ PCR cycles +35 Big Dye cycles)). This estimate represents $1 \%$ of overall nucleotide divergence and will not exceed in any case the $2 \%$ threshold of within-species variation established by the BOLD initiative. PGR costs and sample manipulation can be considerably reduced if the number of engorged arthropods is large enough to make unnecessary the analysis of those specimens containing tiny bloodmeals or bloodmeals into an advanced digestion stage. Direct sequencing methods, which represent $>80 \%$ of overall costs, can be also replaced by other less expensive and more straightforward molecular methods [reviewed in 3] once researchers have accomplished a first preliminary survey using our general approach. The application of our method throughout hotspots of biodiversity is however strongly encouraged given that alternative methods can difficultly provide similar coverage thresholds and sample manipulation convenience.

Importantly, we show that the analysis of sequencing electropherograms could be useful for the identification of mixed bloodmeals from even closely related species (see Fig. 1). The development of specific software to assist the identification of mixed sequencing electropherograms using the sequence data deposited in the public databases must be encouraged in this respect. PCR reactions can be nevertheless highly competitive in mixed bloodmeals and some DNA data could be missing due to partial or total degradation of one of the host's DNA and/or because of the amount of ingested blood from different host species greatly differs. To solve this potential methodological limitation, some authors have satisfactorily used multiplexed primers targeting to different vertebrate groups $[16,17$, but see the multiplexing drawbacks reviewed in 3]. In the very next future, new generation and cost-effective sequencing technologies such as pyrosequencing are expected to revolutionize bloodmeal analyses because it will permit researchers to simultaneously screen a wider array of DNA sequences from mixed samples at different proportions $[18,19]$.

Our method could have a limited value, however, for those species parasitizing known hosts most of the time (e.g. ticks, fleas, mites or lices). Engorged ectoparasites can be nonetheless collected off-hosts using traps or drag sampling [20,21]. Ticks deserve a particular consideration because of their mechanism of blood digestion differs from the rest of blood-feeding arthropods. DNA digestion seems to occur more quickly, and PCR-based methods have proven to vary considerably between different laboratories. Thus, recent research has encouraged the use of tryptic-digestion mass spectrometry of blood proteins to identify various hosts with a single tick bloodmeal [22]. Nevertheless, increased costs and a very limited availability of protein databases for non-model species are two important drawbacks.

In conclusion, we provide a novel, relatively straightforward and cost-effective molecular method that permits researchers to get deeper into the investigation of vector-borne disease ecology and co-evolutionary relationships. The reinforcement of our knowledge about blood-feeding behaviour of haematophagous arthropods and transmission patterns in wild species should decisively contribute to an efficient evaluation and modelling of epidemiologic risks and a better understanding of ecological networks.

\section{Materials and Methods}

\section{Sampling of engorged ectoparasites and study area}

Wildlife engorged mosquitoes, culicoids and sand flies (Class Insecta, Order Diptera, Families Culicidae, Ceratopogonidae and Phlebotomiae) were captured using CDC traps supplied with dry ice to attract ectoparasites through light and $\mathrm{CO}_{2}$. Traps were placed in several locations of South-western Spain, including the Doñana National Park, and the specimens collected the next day. This fact is important given that some studies have shown that the probability of amplifying host DNA after 36 hours from feeding decreases considerably [23]. Engorged ticks (Class Arachnida, Order Acari) were directly sampled from known parasitized hosts (mice and dogs). Individual ectoparasites were identified at the species or genus level. In addition, pathogen-free sucking bugs (Dipetalogaster maximus, Class Insecta, Order Hemiptera, Subfamily Triatominae) used for non-invasive blood sampling [see 24] of captive Iberian lynxs Lynx pardinus (www.lynxexsitu.es), were also obtained. Ectoparasites were preserved at $-80^{\circ} \mathrm{C}$ until DNA extraction. Overall, we sampled 100 engorged mosquitoes plus a few specimens from other families of blood-feeding ectoparasites (Table 1).

\section{DNA extraction}

Individual ectoparasites were processed in Petri plates. Using sterile tips, we pressed deeply on the abdomen of engorged individuals to release bloodmeals. According to the HotSHOT protocol [25], bloodmeals were pipetted into $50 \mu \mathrm{l}$ of lysis solution (25 mM NaOH, $0.2 \mathrm{mM} \mathrm{EDTA)} \mathrm{and} \mathrm{latter} \mathrm{incubated} \mathrm{at} 95^{\circ} \mathrm{C}$ during $30 \mathrm{~min}$. In those cases where bloodmeals could not be easily extracted from the midgut of the ectoparasite, we cut the entire abdomen, which was introduced and crushed into the lysis solution. In addition, at least two negative DNA extraction control (i.e. absence of tissue) were performed during PCR experiments. After incubation, the solution was put on ice for five minutes and then we added $50 \mu \mathrm{l}$ of neutralization solution ( $40 \mathrm{mM}$ Tris- $\mathrm{HCl})$. Bloodmeals or abdomens were simultaneously processed using 96thermowell plates or 8-thermowell individual strips and stored at $-20^{\circ} \mathrm{C}$ until PCR amplification.

\section{Primer design strategy}

We downloaded all vertebrate COI sequences $(\mathrm{N}=18,928)$ from the Classes Mammalia, Aves, Amphibia and Reptilia that were available in the public domain managed by the BOLD Systems database in January 2009 (www.barcodinglife.org). We also downloaded 6,784 arthropod COI sequences from taxonomic groups that included blood-feeding species. This survey included species from the Classes Arachnida (Order Acari) and Insecta (Order Diptera, Families Culicidae, Simuliidae, Ceratopogonidae, Hippoboscidae, 
Tabanidaeand Glossinidae; Order Hemiptera, SubfamiliyTriatominae and Order Phthiraptera). COI sequences were aligned using the software BioEdit 7.0.9.0 [26]. Our multiple alignments revealed several conserved nucleotide positions at the $5^{\prime}$ end of the COI gene. We designed a universal-forward primer with an M13-tail added at the 5' end (M13BC-FW 5'-TGT AAA ACG ACG GCG AGT-HAA YGA YAA RGA YAT YGG NAC-3'), similar to other primers previously used in other barcode approaches [e.g. 13]. Then, we searched for a reverse primer that allowed the specific amplification of vertebrate COI sequences while avoiding the co-amplification of invertebrate DNA (BCV-RV1 5'-GCY GAN AYY ATN GYY RTR (T)(A)-3'). Three nucleotide positions always differing between vertebrate and invertebrate COI sequences are underlined. An additional difference in base composition, which position varies depending on particular arthropod Families, is indicated in brackets. A positional nucleotide numerical summary carried out using BioEdit revealed that the matching of our forward primer to the target sequence was $>99 \%$ at each nucleotide position, after analyzing more than 6,000 COI sequences. The vertebrate-specific reverse primer matched the target sequence with values $>99 \%$ at every nucleotide position, after comparing a minimum number of 5,814 vertebrate COI sequences from the four taxonomic Classes. The mismatching of invertebrate COI sequences with respect to our vertebrate-specific primer was also $>99 \%$ in at least all the four nucleotide positions mentioned above. This estimate was calculated after comparing 3,273 arthropod COI sequences.

\section{PCR amplification and sequencing of vertebrate COI sequences}

The expected length (excluding primers) of the PCR product obtained with primers M13BCV-FW and BCV-RV1 is $758 \mathrm{bp}$. An optimized PCR protocol consisted of $4 \mathrm{~min}$ at $94^{\circ} \mathrm{C}$, followed by 35 cycles of $40 \mathrm{~s}$ at $45^{\circ} \mathrm{C}, 1 \mathrm{~min}$ at $72^{\circ} \mathrm{C}$ and $40 \mathrm{~s}$ at $94^{\circ} \mathrm{C}$, with a final extension step of $7 \mathrm{~min}$ at $72^{\circ} \mathrm{C}$. PCR reactions were carried out using a PTC-100 Programmable Thermal Controller (MJ Research) in a final volume of $30 \mu$ containing 1 unit of a commercial Taq Polymerase (Bioline), 1X manufacturer-supplied buffer (Bioline), $2.5 \mathrm{mM} \mathrm{MgCl} 2,0.25 \mathrm{mM}$ of each dNTP, $5 \%$ DMSO, $10 \mu \mathrm{g}$ of BSA (Bovine Serum Albumin - Amersham corp.), $0.16 \mu \mathrm{M}$ of primers M13BCV-FW and BCV-RV1 and $1 \mu \mathrm{l}$ of extracted DNA. Bloodmeals can be sometimes partially digested, and thus, the number of DNA molecules to be used as templates decreases. Gradual digestion of DNA in arthropod's midgut should be heterogeneous across the sample, and depends on the time elapsed since the ectoparasite has fed. Thus, in order to standardize the number of amplified DNA copies before sequencing, we designed a PCR re-amplification protocol. Further, re-amplification of apparently negative PCR reactions has proven to be successful in previous studies [17]. In this second PCR reaction, we used the M13 primer and a modified reverse primer from that of the first PCR $\left(\mathrm{BCV}-\mathrm{RV} 2=5^{\prime}\right.$-GCY CAN AYY ATN GYY RTR TAN CG-3'). Terminal cytosine nucleotides match to two conserved guanines of the COI locus in eukaryotes. This second PGR reaction consisted of $3 \mathrm{~min}$ at $94^{\circ} \mathrm{C}$ followed by a touch down protocol decreasing the annealing temperature from $60^{\circ} \mathrm{C}$ to $45^{\circ} \mathrm{C}$ during $40 \mathrm{~s}\left(-1^{\circ} \mathrm{C} /\right.$ cycle), with $1 \mathrm{~min}$ extension and $40 \mathrm{~s}$ denaturalization steps at $72^{\circ} \mathrm{C}$ and $94^{\circ} \mathrm{C}$, respectively, followed by 24 cycles of $40 \mathrm{~s}$ at $45^{\circ} \mathrm{C}, 72^{\circ} \mathrm{C}$ and $94^{\circ} \mathrm{C}$, and a final elongation step of $7 \mathrm{~min}$ at $72^{\circ} \mathrm{C}$. PCR reactions were carried out in a final volume of $30 \mu \mathrm{l}$ containing 1 unit of Taq Polymerase (Bioline), 1X manufacturer-supplied buffer, $1.7 \mathrm{mM} \mathrm{MgCl}_{2}, 0.25 \mathrm{mM}$ of each dNTP, 5\% DMSO, $5 \mu \mathrm{g}$ of BSA, $0.16 \mu \mathrm{M}$ of M13 and BCV-RV2 primers and $1 \mu \mathrm{l}$ of the PCR products obtained during the first amplification step (M13BC-FW/BCV-RV1). We used negative controls of PGR amplification (i.e. absence of DNA template) to detect contaminations derived from the presence of bovine (because of the use of BSA) and human DNA. As positive control, we used the bloodmeal of a mosquito sampled while feeding on a mallard duck captured for ringing. A further analysis of repeatability was accomplished by applying the same protocol to a couple of bloodmeals reporting rare hosts (i.e. hosts only found in one bloodmeal). The species used for the repeatability analyses were the crested lark Galerida cristata and the Egyptian mongoose Herpestes ichneumon. The COI locus from both species was amplified and sequenced independently in three different occasions. An identical PCR protocol was applied to DNA extracts obtained from the abdomen of non-engorged mosquitoes (Culex sp., $\mathrm{N}=2$ ), ticks (Hyalomma sp., $\mathrm{N}=2$ ), culicoids (Culicoides sp., $\mathrm{N}=2$ ) and sand flies (Phlebotomus sp., $\mathrm{N}=2$ ) to ensure the unsuccessful amplification of invertebrate DNA. Vertebrate DNA was used during these PCR experiments to control for systematic PGR failure. To identify problems related to the quality of the invertebrate DNA extracts, we performed additional PCR experiments using the eukaryote-universal mini-barcoding primers proposed by Meusnier and co-workers [13]. PCR products were visualized in $1.5 \%$ agarose gels and cleaned-up using the commercial ExoSAP-IT reagent (GE Healthcare Life Sciences). Sequencing reactions were performed according to the BigDye 1.1 technology (Applied Biosystems) using 5 pmoles of the BCV-RV2 primer. This primer is located around $100 \mathrm{bp}$ downstream from the $3^{\prime}$ end of the molecular target for DNA barcoding. Labelled DNA fragments were resolved using an ABI 3130xl automated sequencer (Applied Biosystems).

\section{Identifying unambiguous and ambiguous unknown COI sequences}

We used the identification engine implemented in the BOLDIDS platform (http://www.barcodinglife.org/views/idrequest. php) to assign unknown COI sequences to particular species. The BOLD-IDS component provides a species identification tool that accepts DNA sequences from the barcode region and returns a taxonomic assignment to the species level when possible. We always chose to search within the reference barcode dataset (i.e. validated subset of the full database with a minimum sequence length of $500 \mathrm{bp}$ and containing only those species represented by three or more individuals showing less than $2 \%$ sequence divergence) when possible.

Additionally, haematophagous arthropods can feed on several hosts. If hosts belong to different species, the resulting sequencing electropherogram will contain a variable number of ambiguities (i.e. double peaks). We used BioEdit to introduce IUPAC degenerate codes (i.e. those representing different sets of nucleotides) across sequencing electropherograms. Ambiguous COI sequences were aligned with respect to a reference data set containing unambiguous COI sequences from the vertebrate species identified in our study area so far. Then, we removed from the alignment those COI sequences differing from the ambiguous unknown sequence in at least $2 \%$ of nucleotide positions (i.e. the within-species threshold proposed by the DNA barcoding initiative) until only a few candidate species remained.

\section{Acknowledgments}

We are very grateful to Astrid Vargas, from the Ex-situ Conservation Program of the Iberian Lynx (www.lynxexsitu.es), and to the Institute of Wildlife and Zoological Research (IZW, Berlin, Germany, www.izw-berlin. 
de) for kindly providing blood-fed specimens of Dipetalogaster maximum. We also thank JL Barroso for providing engorged ticks and Esmeralda Pérez for her work collecting and classifying mosquito. We thank to Dr. Ng LeeChing for comments and suggestions on an earlier version of the manuscript.

\section{References}

1. Jones KE, Patel NG, Levy MA, Storeyard A, Balk D, et al. (2008) Global trends in emerging infectious diseases. Nature 451: 990-994.

2. Gratz NG (1999) Emerging and resurging vector-borne diseases. Annu Rev Entomol 44: 51-75.

3. Kent RJ (2009) Molecular methods for arthropod bloodmeal identification and applications to ecological and vector-borne disease studies. Mol Ecol Res 9: 4-18.

4. Ratnasingham S, Hebert PDN (2007) BOLD: The Barcode of Life Data System. Mol Ecol Notes 7: 355-364.

5. Waugh J (2007) DNA barcoding in animal species: progress, potential and pitfalls. BioEssays 29: 188-197.

6. Valentini A, Pompanon F, Taberlet P (2008) DNA barcoding for ecologists. Trends Ecol Evol 24: 110-117.

7. Hebert PDN, Cywinska A, Ball SL, deWaad JR (2003) Biological identifications through DNA barcodes. Proc R Soc B 270: 313-321.

8. Tavares ES, Baker JA (2008) Single mitochondrial gene barcodes reliably identify sister-species in diverse clades of birds. BMC EvolBiol 8: 81.

9. Wheeler SS, Barker CM, Fang Y, Arnijos MV, Carrol BD, et al. (2009) Differential impact of West Nile Virus on California Birds. The Condor 111: $1-20$.

10. Pérez-Tris J, Bensch S (2005) Dispersal increases local transmission of avian malarial parasites. Ecology Letters 8: 838-845.

11. Foley JE, Clueit SB, Brown RN (2008) Differential exposure to an aplasmaphagocytophilum in rodent species in northern California. Vector Borne Zoonitc Dis 8: 49-55.

12. Townzen JS, Brower AVZ, Judd DD (2008) Identification of mosquito bloodmeals using mitochondrial cytochrome oxidase subunit I and cytochrome b gene sequences. Med Vet Entomol 22: 386-393.

13. Meusnier I, Singer GAC, Landry JF, Hickey DA, Hebert PD, et al. (2008) A universal DNA mini-barcode for biodiversity analyses. BMC Genomics 9: 214.

14. Kocher TD, Thomas WK, Meyer A, Edwards SV, Pääbo S, et al. (1989) Dynamics of mitochondrial DNA evolution in animals: amplification and sequencing with conserved primers. Proc Natl Acad Sci USA 86: 6196-6200.

\section{Author Contributions}

Conceived and designed the experiments: MA CR SR RS JF. Performed the experiments: MA. Analyzed the data: MA SR. Contributed reagents/ materials/analysis tools: MA SR JM RS JF. Wrote the paper: MA CR JM JF.

15. Oshaghi MA, Chavshin AR, Vatandoost H, Yaaghoobi F, Mohtarami F, et al. (2006) Effects of post-ingestion and physical conditions on PCR amplification of host blood meal DNA in mosquitoes. ExperimParasitol 112: 232-236.

16. Kent RJ, Norris DE (2005) Identification of mammalian blood meals of mosquitoes by a multiplexed polymerase chain reaction targeting cytochrome $b$. Am J Trop Med Hyg 73: 336-342.

17. Kent RJ, West AJ, Norris DE (2004) Molecular differentiation of colonized human malaria vectors by $28 \mathrm{~S}$ rDNA polymorphisms. Am J Trop Med Hyg 71: 514-51.

18. King RA, Read DS, Traugott M, Symondson OC (2008) Molecular analysis of predation: a review of best practice for DNA-based approaches. Mol Ecol 17: 947-963.

19. Traugott M, Bell JR, Broad GR, Powel W, Van Been FJF, et al. (2008) Endoparasitism in cereal aphids: molecular analysis of a whole parasitoid community. Mol Ecol 17: 3928-3938.

20. Woods MR, Montenieri JA, Eisen RJ, Zeidner NS, Borchert JN, et al. (2009) Identification of Flea Blood Meals Using Multiplexed Real-Time Polymerase Chain Reaction Targeting Mitochondrial Gene Fragments. Am J Trop Med Hyg 80: 998-1003.

21. Falco RC, Fish D (1992) A comparison of methods for sampling the deer tick, Ixodesdammini, in a Lyme disease endemic area. Exp Appl Acarol 14: 165-173.

22. Wickramasekara S, Bunikis J, Wysocki V, Barbour AG (2008) Identification of Residual Blood Proteins in Ticks by Mass Spectrometry Proteomics. Emerg Infect Dis 14: 1273-1275.

23. Rasgon J (2008) Stable isotope analysis can potentially identify completelydigested bloodmeals in mosquitoes. PLoS ONE 3: 1-3.

24. Thomsen R, Voigt CC (2006) Non-invasive blood sampling from primates using laboratory-bred blood-sucking bugs (Dipetalogaste rmaximus; Reduviidae, Heteroptera). Primates 47: 397-400.

25. Truett GE, Walker JA, Truett AA, Mynatt RL, Heeger P, et al. (2000) Preparation of PCR-quality DNA with hot sodium hydroxide and tris (HotSHOT). Biotechniques 29: 52-54.

26. Hall TA (1999) BioEdit: a user-friendly biological sequence alignment editor and analysis program for Windows 95/98/NT. Nucleic Acids Symp Ser 41: 95-98. 